\title{
Impact of Surface Roughness on the Turbulent Wake Flow of a Turbine Blade
}

\author{
K. Mulleners, P. Gilge, and S. Hohenstein \\ Institute of Turbomachinery and Fluid Dynamics, Leibniz Universität Hannover, Appelstrasse 9, 30167 Hannover, Germany \\ Correspondence should be addressed to K. Mulleners; mulleners@tfd.uni-hannover.de
}

Received 4 September 2014; Accepted 9 December 2014; Published 30 December 2014

Academic Editor: Noor A. Ahmed

Copyright (C) 2014 K. Mulleners et al. This is an open access article distributed under the Creative Commons Attribution License, which permits unrestricted use, distribution, and reproduction in any medium, provided the original work is properly cited.

Roughened aeroengine blade surfaces lead to increased friction losses and reduced efficiency of the individual blades. The surface roughness also affects the wake flow of the blade and thus the inflow conditions for the subsequent compressor or turbine stage. To investigate the impact of surface roughness on a turbulent blade wake, we conducted velocity field measurements by means of stereo particle image velocimetry in the wake of a roughened turbine blade in a linear cascade wind tunnel. The turbine blade was roughened at different chordwise locations. The influence of the chordwise location of the added surface roughness was examined by comparing their impact on the width and depth of the wake and, the positions and distribution of vortical structures in the wake. Additionally, the friction loss coefficients for different surface roughness positions were estimated directly from the velocity field.

\section{Introduction}

The aerodynamics of multistage turbomachinery is extremely complex due to the inherent unsteadiness and threedimensionality of the internal flow. Designing aerodynamically efficient aeroengines is a challenging task that requires detailed knowledge about unsteady boundary development and transition $[1,2]$, the structure of blade wakes $[3,4]$, and the interaction of these wakes with downstream blades $[5,6]$. The dominating unsteadiness of the internal engine flow results from the relative motion of rotor and stator blades and is periodic in nature. Additionally, turbulent inflow conditions and variations of the blades' geometry and surface roughness within a turbine or compressor stage lead to aperiodic fluctuations of the flow. Although the stochastic fluctuations are generally smaller in amplitude than the periodic variations, they can have significant influence on the aerodynamic $[7,8]$, aeroacoustic [9], and aeroelastic properties $[10,11]$ of turbines and compressors.

Surface roughness on aeroengine blades can be induced by manufacturing or repair processes and by operational and environmental conditions. It reduces the blades' efficiency by increasing the boundary layer momentum loss and blade skin friction and by precipitating boundary layer transition.
At high Reynolds numbers, surface roughness also raises the odds for flow separation to occur, leading to further increased losses. The influence of surface roughness on a particular blade does not only affect that blade's efficiency, but also reaches even further by affecting the wake flow and thus the inflow conditions for subsequent compressor or turbines stages.

To allow for economical manufacturing and repair processes and to improve and tailor the individual process steps, geometric and dimensional manufacturing tolerances based on the aerodynamic effects of surface roughness are required. Determining these tolerances requires a profound understanding of the effect of surface roughness on the aerodynamic performance of aeroengine blades, including secondary effects on the turbulent wake flow.

To investigate the impact of surface roughness on a turbulent blade wake, we conducted velocity field measurements in the wake of a roughened turbine blade in a linear cascade wind tunnel by means of stereo particle image velocimetry (PIV). The turbine blade was roughened at different chordwise locations considering two roughness heights.

In the following, the turbine blade profile, surface roughness application, and experimental setup are described first. 


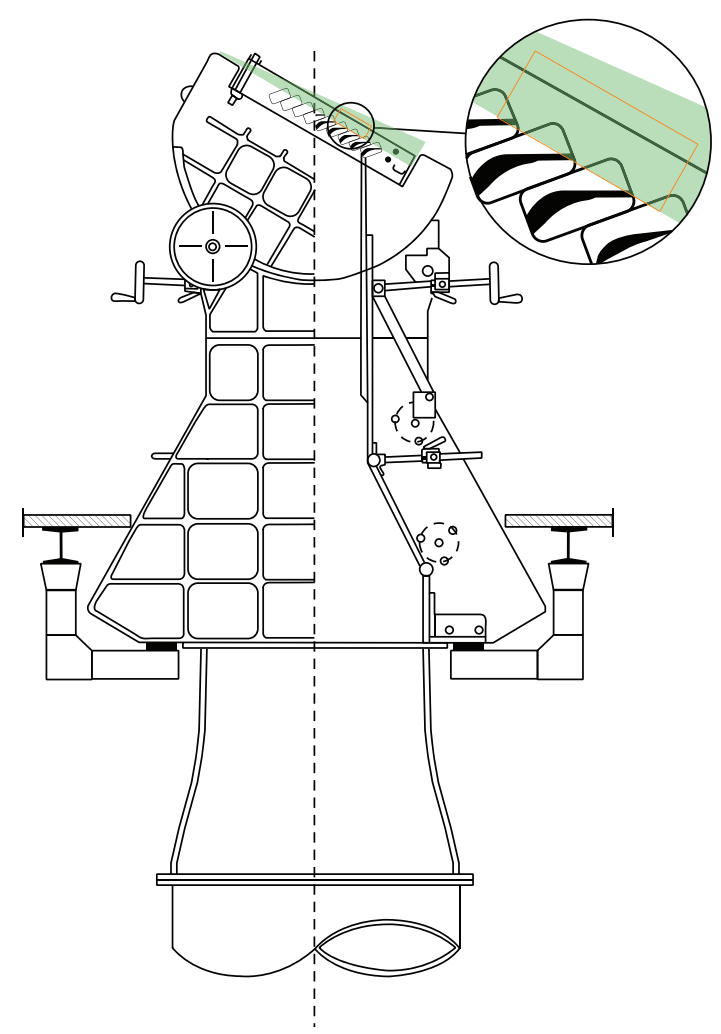

FIGURE 1: The linear cascade wind tunnel at the Institute of Turbomachinery and Fluid Dynamics (Hannover, Germany). The PIV region of interest is presented in the inset.

Subsequently, the experimental results are presented and discussed focusing on the impact of the added surface roughness on the width and depth of the wake, the positions, and distribution of vortical structures. A recently proposed method to estimate the friction loss coefficient directly from the measured velocity field data is presented and the variation of the friction loss with added surface roughness is addressed.

\section{Materials and Methods}

2.1. Linear Cascade Wind Tunnel. Velocity field measurements by means of stereo PIV were conducted in a cross sectional plane in the wake of turbine blades in the linear cascade wind tunnel of the Institute of Turbomachinery and Fluid Dynamics of the Leibniz Universität Hannover (Figure 1). The air flow in the linear cascade wind tunnel was supplied by three screw-type air compressors allowing for a maximum mass flow of $8.9 \mathrm{~kg} / \mathrm{s}$. Compressed air was cooled down to a temperature of $298 \mathrm{~K} \pm 5 \mathrm{~K}$ and guided through flow straighteners and a settling chamber to generate a homogeneous inflow with a turbulence intensity of $4.5 \%$ in the cascade inlet plane. The position of the cascade box can be progressively varied to alter the inflow angle. A boundary layer suction system at the side walls of the cascade box prevents flow separation at the blade corners ensuring twodimensional flow conditions.

2.2. Turbine Blade Profiles and Surface Roughness. The wind tunnel blades were designed to meet the aerodynamic properties of turbine blades in a second stage of a high pressure turbine of a civil aircraft engine [12]. The cascade box contained seven blades in total to ensure periodic flow conditions. The middle blade was split into two parts, where one part served as a reference state and the other part was roughened in the present investigation, similar to the procedure followed by Lietmeyer [13] (Figure 2(a)). Surface roughness was applied on the suction side of the blade at four positions along the chord: $0.05 c, 0.2 c, 0.5 c$, and $0.85 c$ (Figure 2(b)). This corresponds to the very leading edge, the position where the streamwise pressure gradient changes sign, and positions directly before and after boundary layer transition. The applied surface roughness includes spanwise bands of $4 \mathrm{~mm}$ width covered with sand grains of mesh sizes 120 and 80 at the prescribed location.

2.3. PIV Setup. The velocity field in the wake of the roughened turbine blade was measured for two different inflow Mach numbers, $\mathrm{Ma} \in\{0.10,0.15\}$ and normalised roughness heights $k / c \in\{0.02,0.03\}$. The Reynolds numbers based on the outflow conditions are $\mathrm{Re}=211600$ and 322000 , spanning the range of expected Reynolds numbers of aircraft engines in cruise conditions [14].

The stereoscopic PIV data was evaluated according to standard procedures (cf. Raffel et al. [15]) using the multigrid algorithm with image deformation and a final interrogation window size of $24 \mathrm{px} \times 24 \mathrm{px}$ and an overlap of $50 \%$. This yields a grid spacing or physical resolution of $12 \mathrm{px}$ or $1.33 \mathrm{~mm}=$ $0.02 c$. Series of thousand images were recorded for different 


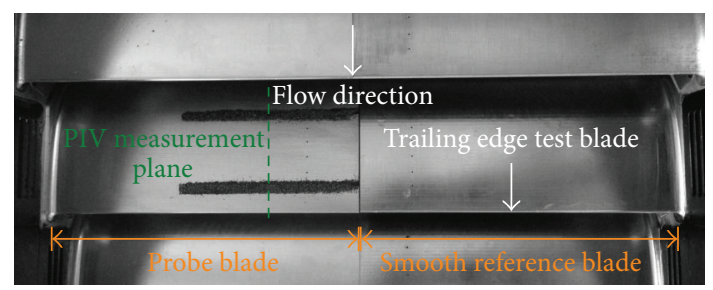

(a)

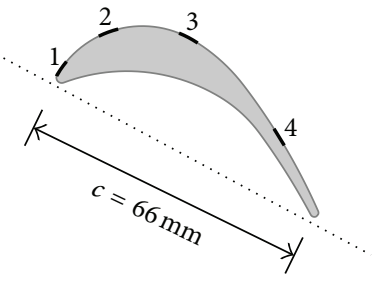

(b)

Figure 2: Top view of the test blade in the linear cascade wind tunnel (a) and turbine blade profile with chord length $c$ (b). On locations 1-4 the surface is successively roughened along part of the blade height on the probe blade.

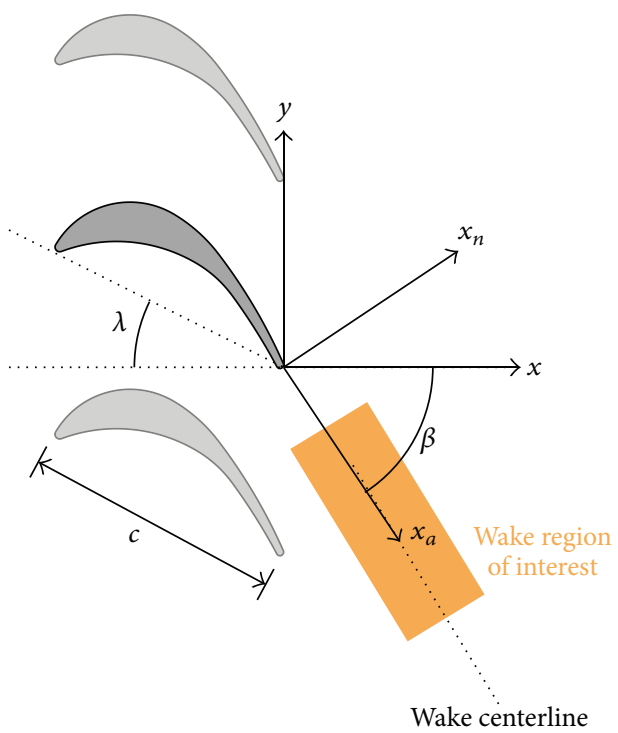

FIGURE 3: Definition of the two coordinate systems in which the data is presented. Additionally, the blade's chord length $c$, blade setting angle $\lambda$, and blade wake angle $\beta$ are indicated.

surface roughness application and different inflow Mach numbers to ensure convergence of the first- and second-order statistical properties.

The probability for detecting valid displacements in the PIV data set was higher than $90 \%$ and remaining spurious vectors were detected and replaced using the automated postprocessing algorithm of Garcia [16]. The inaccuracy in determining the valid displacements is given by the single displacement error, which is decomposed into the random error or measurement uncertainty and the bias error. Based on careful analysis of the results, the random error was estimated smaller than $0.2 \mathrm{px}$ and the bias error smaller than $0.05 \mathrm{px}$.

The data is presented in two coordinate systems (Figure 3). The $(x, y)$-coordinate system is fixed in the linear cascade tunnel reference system where $x$ and $y$ represent the axial and vertical coordinates. The origin coincides with the trailing edge of the blade under investigation. To facilitate comparison of the wake parameters, part of the data is presented in a wake-oriented coordinate system $\left(x_{a}, x_{n}\right)$, with $x_{a}$ being the streamwise coordinate along the wake centerline and $x_{n}$ being the wake normal coordinate and the origin still located at the blade trailing edge. The wake centerline is determined by least square fitting locations of minimum time averaged velocity in the wake.

\section{Results and Discussion}

Measured averaged velocity fields in the wake of the turbine blades in a linear cascade are presented in Figure 4 for successively added surface roughness on the suction side of the central cascade blade and $\operatorname{Re}=3.2 \times 10^{5}$. The blade wakes are readily identified by the velocity deficit. With increasing surface roughness the velocity deficit in the wake increases and a clear difference arises between the wakes of the roughened central blade and those of the surrounding untreated smooth blades.

To qualitatively analyse the effect of the localised surface roughness on the wake flow, wake parameters including wake width and blade deflection angle were determined and will be compared here.

3.1. Wake Parameters. As a first step towards the analysis of the wake parameters, the wake centerline of the test blade is determined as the best fitting linear curve connecting 


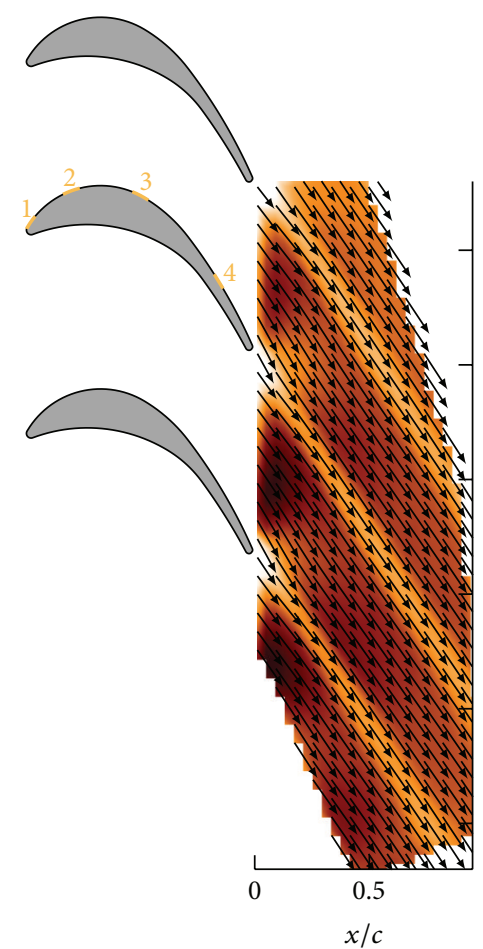

(a)

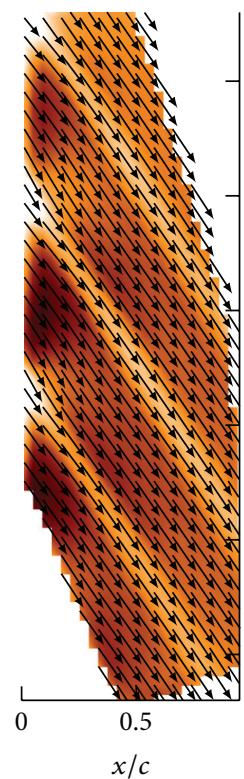

(b)

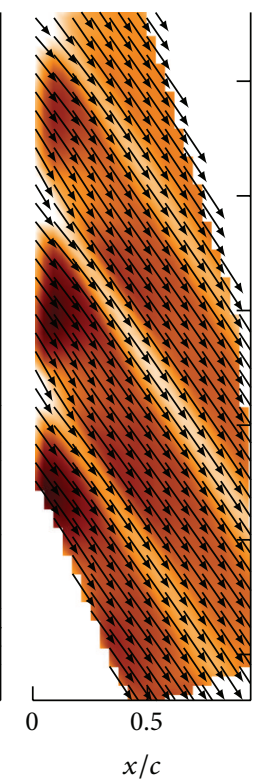

(c)

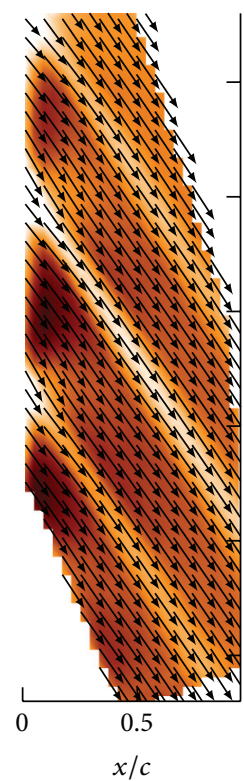

(d)

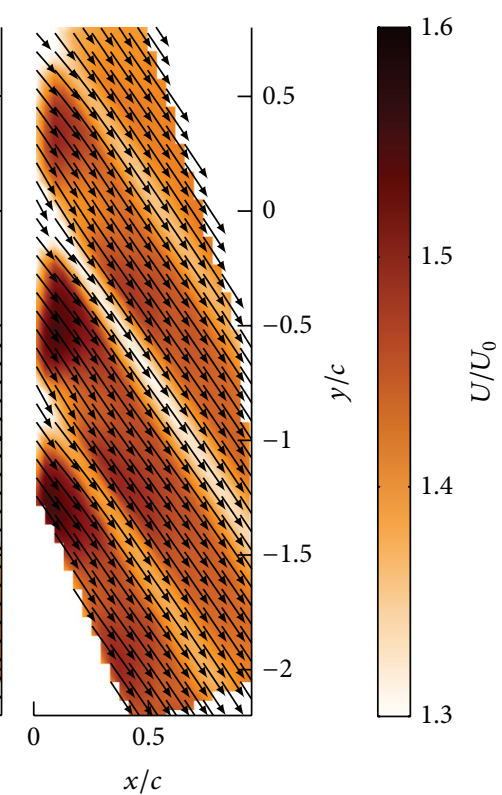

(e)

FIGURE 4: Averaged velocity fields in the wake of the smooth (a) and roughened (b-e) surface blades for Re $=3.2 \times 10^{5}$. The roughness is successively added at indicated locations $1-4$ resulting in the fields $(b-e)$, respectively.

the locations of minimum average velocity in its wake (Figure 5). The angle between the wake centerline and the horizontal represents the deflection of the flow by the blade and is denoted by the wake deflection angle $\beta$. The wake centres are determined with a subgrid resolution. Therefore, the accuracy of the determined value for $\beta$ is mainly determined by the quality of the linear fit. After careful analysis of the results, the uncertainty for $\beta$ was estimated to be smaller than $0.05^{\circ}$.

Presenting the data in the wake-oriented coordinate system (Figures 6(a) and 6(b)) reveals that the out-ofplane vorticity component $\omega_{z}$, defined as $\omega_{z}=\partial v / \partial x-$ $\partial u / \partial y$, vanishes along the wake centerline. This indicates that the wake centerline is well captured by the linear fitting curve based on the velocity minima. The wake width $\Delta$ is determined along the wake centerline as the distance between locations of minimum and maximum vorticities from the cross wake profiles (Figure 6(d)).

The influence of the Reynolds number and the location of surface roughness application on the velocity minimum at wake centerline and the blade deflection angle is summarised in Figure 7. The velocity minimum is a measure for the velocity deficit in the wake and for the aerodynamic loss. With added surface roughness, the minimum velocity decreases, indicating an increase in drag. For $\mathrm{Re}=3.2 \times 10^{5}$, there is a constant decrease with added surface roughness whereas for $\operatorname{Re}=2.1 \times 10^{5}$ the roughness added at the very leading edge does not have a significant influence for $k / c=0.02$. For the lower Reynolds number the boundary layer at leading edge is less susceptible for surface roughness.

A similar behaviour is observed for blade deflection angle. For $\operatorname{Re}=2.1 \times 10^{5}$ and $k / c=0.02$, surface roughness at very leading edge does not yield a measurable change for $\beta$. Once surface roughness is added at location 2 , the blade angle decreases. For $\operatorname{Re}=3.2 \times 10^{5}$, the blade angle is always smaller than for Re $=2.1 \times 10^{5}$ and decreases steadily with added roughness.

Variations of the blade deflection angle are important due to their potential influence on the aeroelastic and aeroacoustic properties of subsequent turbine stages. The influence of the surface roughness height is negligible for the roughness heights considered here and is omitted in the following discussion.

As expected and indicated in Figure 6, the wake width increases along the wake coordinate $x_{a}$ (Figure 8). This evolution is approximately linear for all surface conditions and a constant wake growth rate was determined as the slope of the linear fitting curve. Comparison of the wake growth rate in wake for different surface conditions in Figure 9 reveals that the wake growth rate increases continuously when surface roughness is added to location 2 and beyond. The growth rate is larger for higher Re-number, except for the smooth blade where differences lie within the range of accuracy.

3.2. Direct Estimate of the Loss Coefficient. To quantify the friction loss due to additional surface roughness we applied 


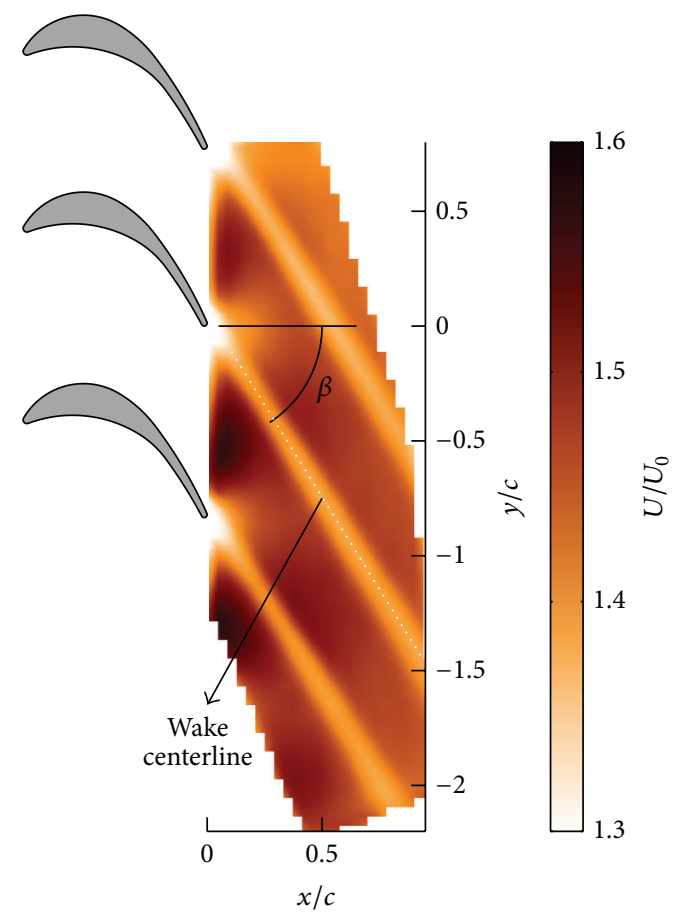

Figure 5: Averaged velocity field in the wake of the smooth surface blade for $\mathrm{Re}=3.2 \times 10^{5}$. The wake centerline is determined as the least square fit of the minimum averaged velocity in the wake.

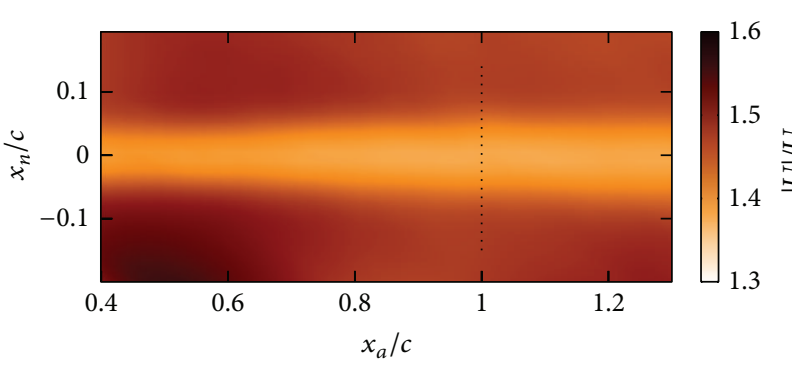

(a)

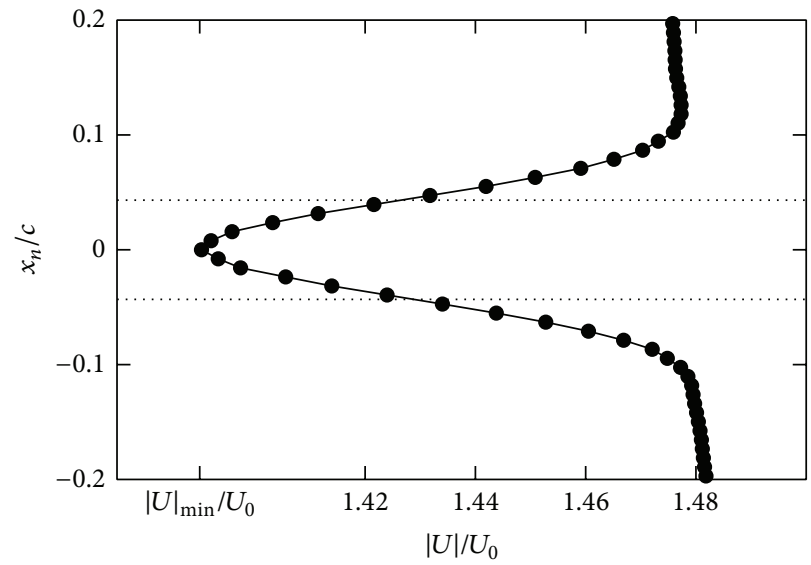

(c)

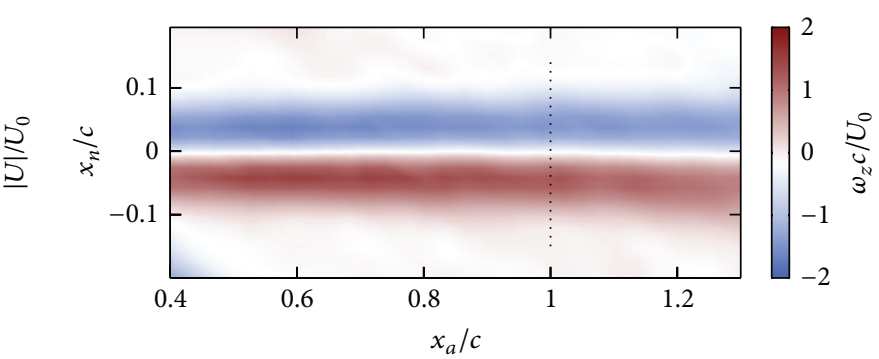

(b)

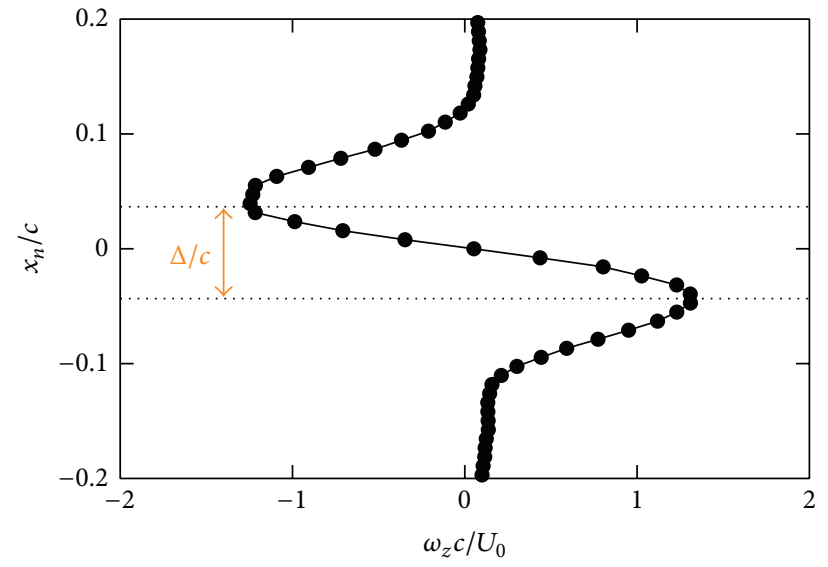

(d)

Figure 6: Averaged velocity (a) and vorticity (b) field in the wake of the smooth surface blade for $\operatorname{Re}=3.2 \times 10^{5}$ represented in the wakeoriented coordinate system. The wake width $\Delta$, determined from cross wake vorticity profiles, is indicated in the cross wake velocity (c) and vorticity (d) profiles one chord length downstream of the blade's trailing edge. 


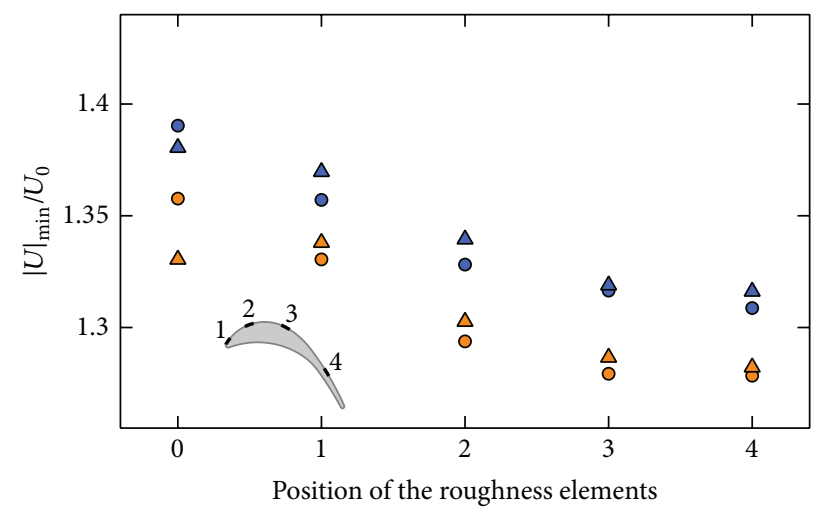

$\Delta \mathrm{Re}=2.1 \times 10^{5}, k / c=0.02 \quad \Delta \mathrm{Re}=3.2 \times 10^{5}, k / c=0.02$

○ $\operatorname{Re}=2.1 \times 10^{5}, k / c=0.03 \quad$ O $\operatorname{Re}=3.2 \times 10^{5}, k / c=0.03$

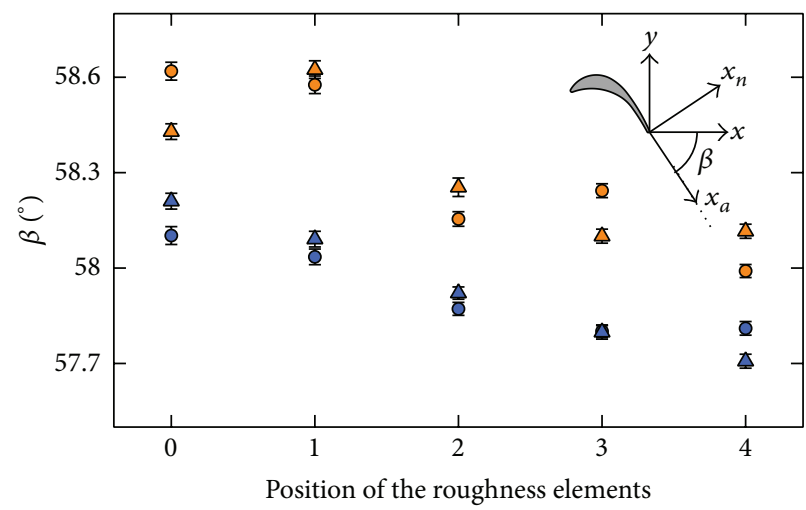

$\Delta \mathrm{Re}=2.1 \times 10^{5}, k / c=0.02 \quad \Delta \mathrm{Re}=3.2 \times 10^{5}, k / c=0.02$

○ $\mathrm{Re}=2.1 \times 10^{5}, k / c=0.03 \quad$ ○ $\mathrm{Re}=3.2 \times 10^{5}, k / c=0.03$

FIGURE 7: Influence of the localised surface roughness on the velocity deficit at $x_{a}=c$ and the wake deflection angle for two Re numbers and two roughness heights.

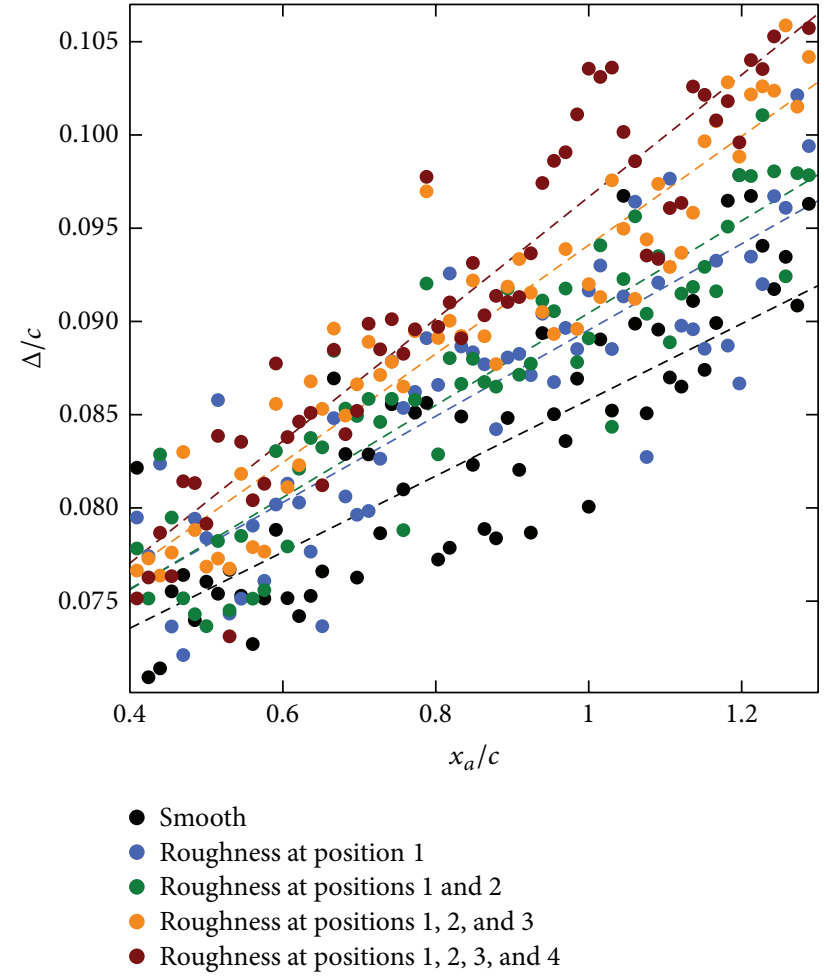

Figure 8: Downstream evolution of the wake width for different applications of surface roughness and $\operatorname{Re}=3.2 \times 10^{5}$ and $k / c=0.03$. The dashed lines represent linear fits of the wake growth.

the method described by Zhang et al. [17], which allows us to estimate loss behaviour directly from the measured velocity field in the wake. According to Zhang et al., the comparative control volume analysis for a smooth and roughened turbine blade can be reduced to

$$
\begin{gathered}
\left.\int_{A_{\text {out }}} \rho_{\text {out }} u_{\text {out }}^{2} d A\right|_{\text {smooth }}-\left.\int_{A_{\text {out }}} \rho_{\text {out }} u_{\text {out }}^{2} d A\right|_{\text {rough }} \\
=F_{s, \text { rough }}-F_{s, \text { smooth }}=\Delta F_{s}
\end{gathered}
$$

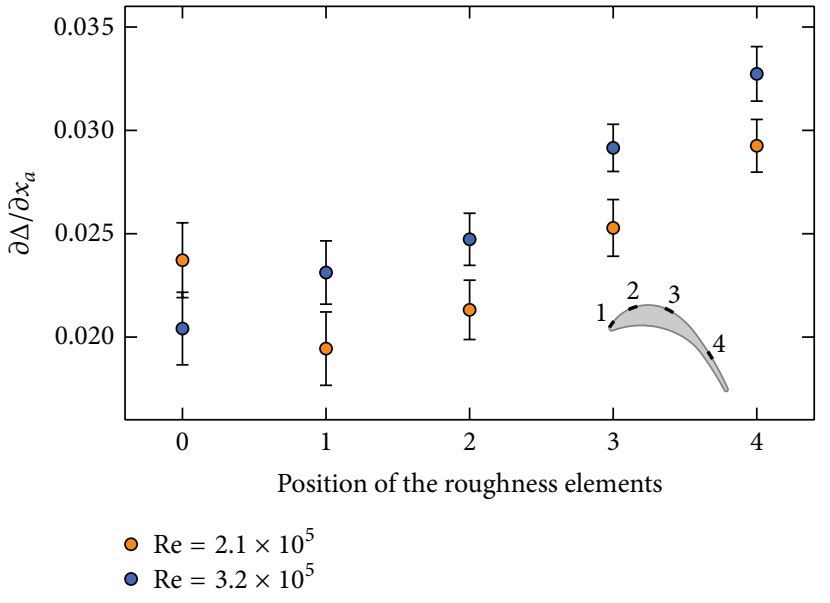

FIGURE 9: Influence of the localised surface roughness on the wake growth rate for two Re numbers and $k / c=0.03$.

with $\rho_{\text {out }}$ and $u_{\text {out }}$ being the density and velocity at the outlet plane $A_{\text {out }}$ of the control volume in Figure 10, under the assumption that variations in pressure force can be neglected with respect to the contributions by the friction force $F_{s}$.

Consequently, a measure of the friction loss due to surface roughness can be determined in terms of a loss coefficient $\zeta_{F_{s}}$, which is defined here as

$$
\zeta_{F_{s}}=\frac{\Delta F_{s}}{\max \left(\Delta F_{s}\right)}
$$

and was calculated by subtracting the momentum flux at $x_{a} / c=1$ corresponding to the smooth and rough surfaced blade.

To compensate for fluctuations of the inflow and minor measurement uncertainties, the wake velocity profiles of the rough surface cases were shifted over an offset $\Delta U_{0}=$ $U_{0, \text { smooth }}-U_{0 \text {,rough }}$ in the outer wake as indicated in Figure 11.

The resulting estimates for the loss coefficient $\zeta_{F_{s}}$ for $\mathrm{Re}=$ $3.2 \times 10^{5}$ are depicted in Figure 12. Despite the negative value 


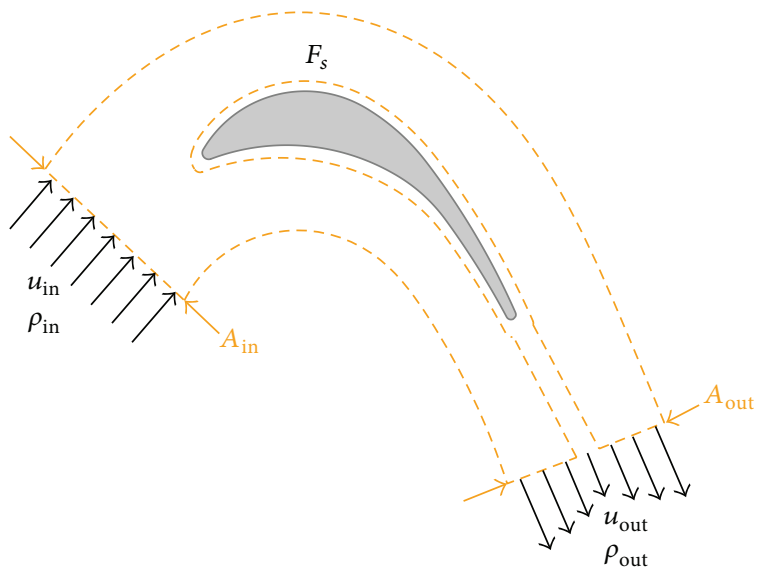

Figure 10: Control volume around the turbine blade.

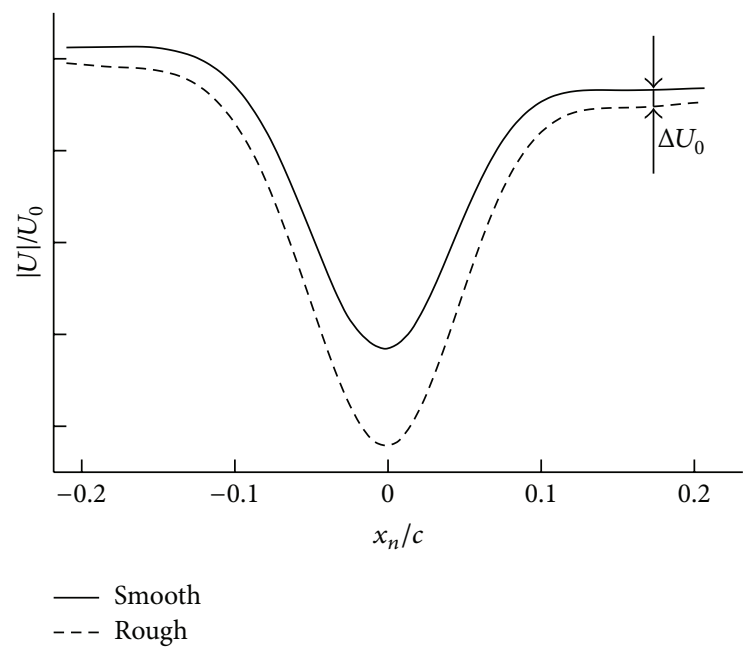

Figure 11: Correction of the wake velocity profiles.

for the resulting loss coefficient corresponding to the surface roughness near the very leading edge, the trend observed for additionally added surface roughness at positions 2, 3, and 4 corroborates previous results. The losses increase with added roughness but in the increase in friction loss decreases. The largest change is observed when roughness is added to positions close to the location of boundary layer transition while additional roughness near the trailing edge only leads to a minor increase of friction losses.

3.3. Wake Vortical Structures. To better understand the continuous increase in wake growth rate in Figure 9 we determine the location of vortical structures in individual snapshot of the wake flow field and subsequently determined the probability distribution of vortical structures (Figure 13). The position of individual vortex cores was detected by using a scalar function $\Gamma_{2}$ introduced by Graftieaux et al. [18] as described previously by Mulleners and Raffel [19].

We notice two things from the probability density maps. First, the probability density for both clockwisely rotating vortices, which are found in the suction side of the wake,

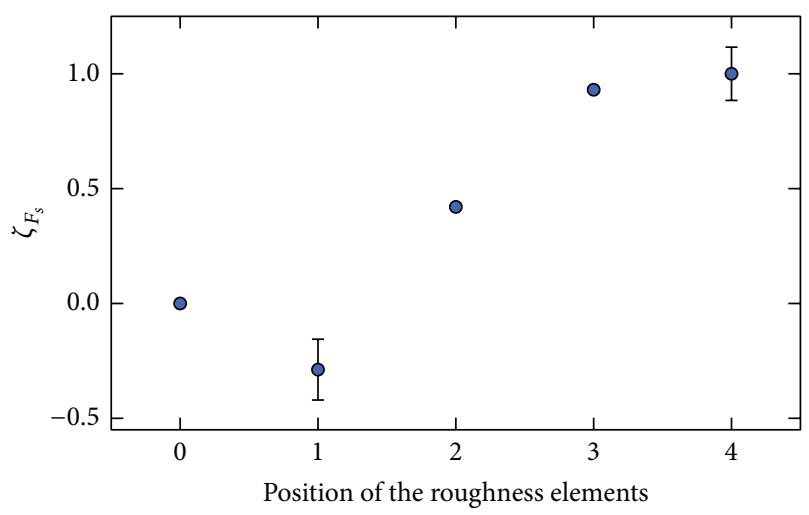

FIGURE 12: Influence of the localised surface roughness on the loss coefficient for $\operatorname{Re}=3.2 \times 10^{5}$ and $k / c=0.03$ according to (1) and (2).

is always larger than that for counterclockwisely rotating vortices, which are encountered in pressure side of the wake. Second, the probability densities for both clockwisely and counterclockwisely rotating vortices increase with added surface roughness.

For the smooth surface, the larger velocity gradients in the suction side boundary layer lead to increased production of clockwisely rotating vortical structures. When surface roughness is added, the velocity in the wake centerline decreases yielding larger shear rates on both sides of the wake, increasing the overall production of vortices. As a result of the increased density of vortical structures at both sides of the wake for successively added roughness, the overall repulsion between clockwisely and counterclockwisely rotating vortices increases leading to the observed increase in wake growth rate.

\section{Conclusion and Perspectives}

Velocity field measurements by means of stereo PIV were conducted in the wake of a locally roughened turbine blade in a linear cascade wind tunnel to investigate the impact of surface roughness on the wake flow. The data was presented in a wake coordinate system to facilitate data analysis. Wake parameters, such as velocity minimum, wake width, and wake angle, were determined and compared for different surface roughness conditions. The position where the surface roughness is applied has a very strong influence on the velocity deficit and wake deflection angle. The wake growth rate was found to increase with added surface roughness, which was related to an increased production and density of vortical structures on both sides of the wake.

Further investigations will explore the impact of threedimensional multiscale roughness topographies representing actual damaged surfaces of engine blades. Due to the inherent complex and three-dimensional nature of multiscale roughness topographies and the wake flows, an experimental approach based on PIV is preferential. To describe the surface topographies and obtain generalisable results, the use of probabilistic methods is desired. Based on the influence 

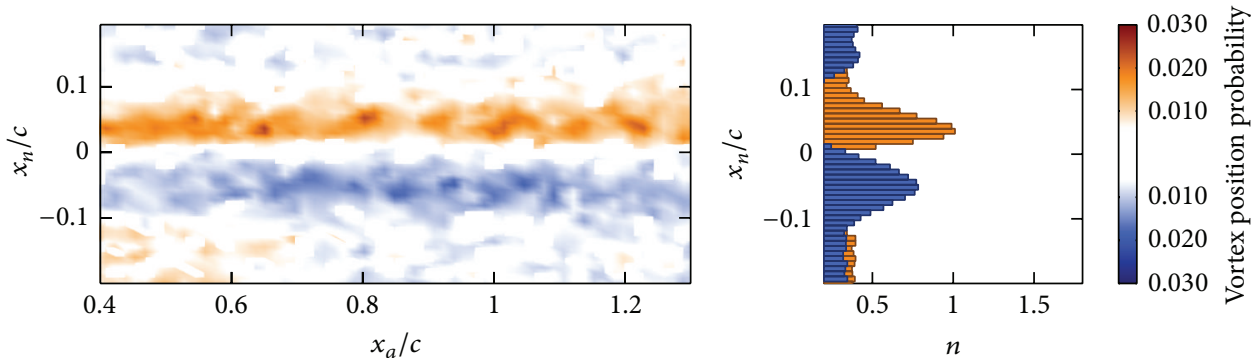

(a)
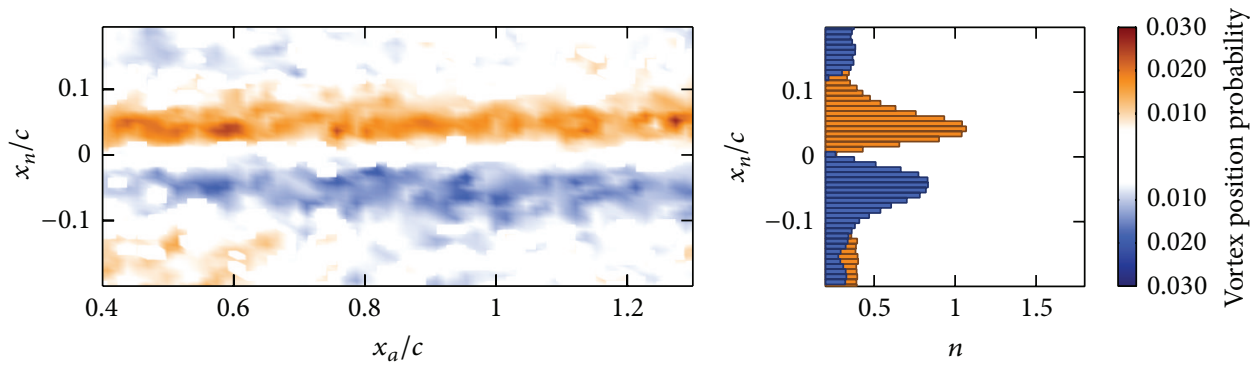

(b)
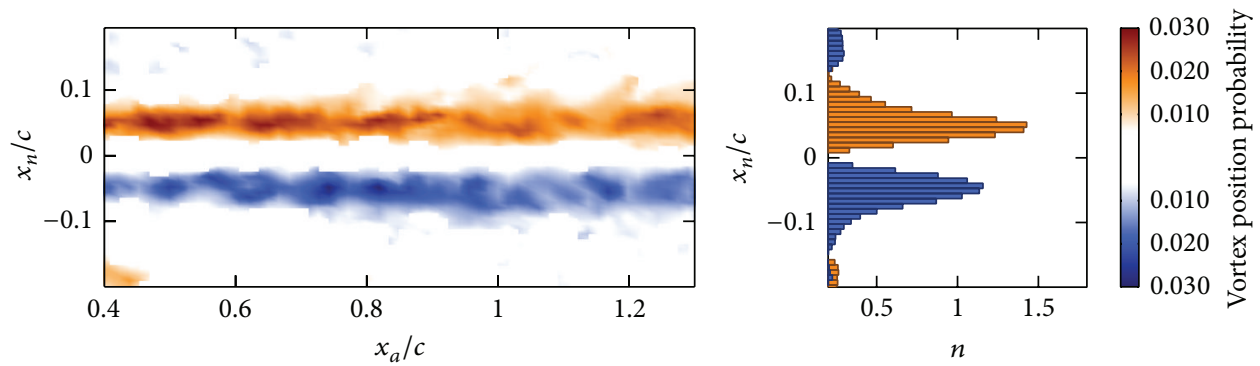

(c)
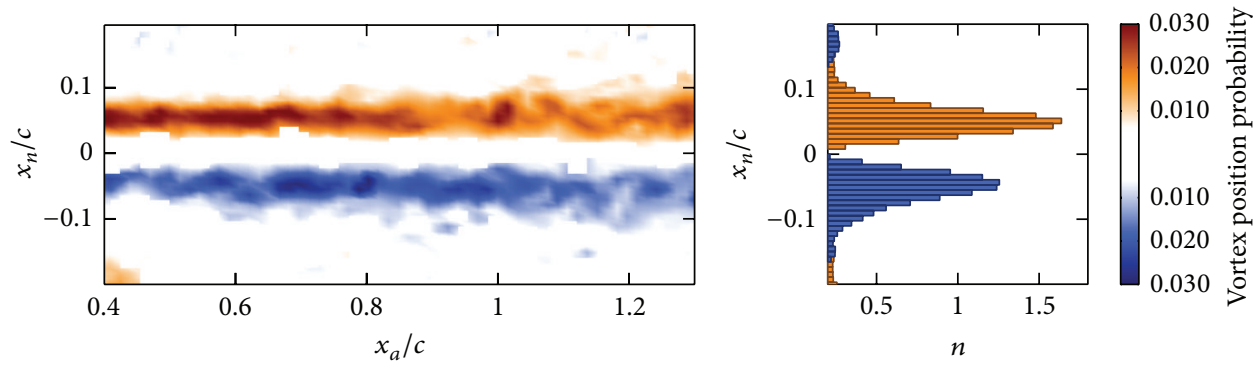

(d)
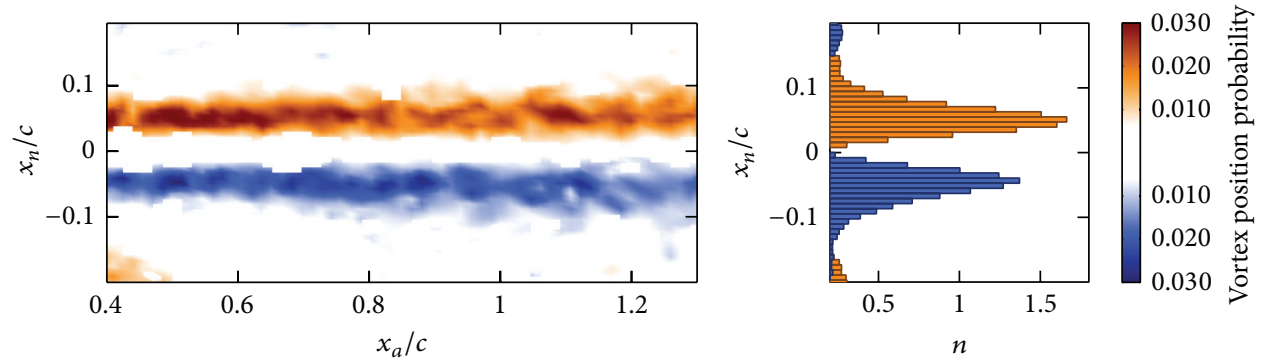

(e)

Figure 13: Probability density of vortical structures in the the wake of the smooth (a) and roughened (b-e) surface blades for Re $=3.2 \times 10^{5}$ and $k / c=0.03$, with (b-e) corresponding to successively added roughness at locations 1-4 (Figure 2). The probabilities along the wake coordinate are summed up in the bar plots at the right. The probability densities of counterclockwisely and clockwisely rotating vortices are associated with shades of blue and red, respectively. 
of multiscale roughness topographies, guidelines for manufacturing and repair processes of aeroengine blades are envisaged.

\section{List of Symbols}

$\begin{array}{ll}\beta: & \text { Wake deflection angle } \\ \gamma: & \text { Setting angle } \\ \Delta: & \text { Wake width } \\ \omega_{z}: & \text { Sut-of-plane vorticity component } \\ k: & \text { Blade chord length } \\ c: & \text { Mach number } \\ \text { Ma: } & \text { Vortex position density } \\ n: & \text { Reynolds number } \\ \text { Re: } & \text { Velocity magnitude } \\ U: & \text { Inflow velocity magnitude } \\ U_{0}: & \text { Velocity deficit at the wake centerline } \\ U_{\text {min }}: & \text { Spatial coordinate system } \\ \vec{x}=(x, y, z): & \text { Wake coordinate system } \\ \vec{x}=\left(x_{a}, x_{n}, x_{z}\right): & \text { Density } \\ \rho: & \text { Wall shear stress force } \\ F_{s}: & \text { Control volume wall force } \\ F_{w}: & \text { Airfoil form drag force } \\ F_{p}: & \text { Loss coefficient. } \\ \zeta_{F_{s}}: & \end{array}$

\section{Conflict of Interests}

The authors declare that there is no conflict of interests regarding the publication of this paper.

\section{Acknowledgment}

Part of this work has been conducted within the framework of subproject B3 entitled Influence of Complex Surface Structures on the Aerodynamic Loss Behaviour of Blades of the Collaborative Research Centre (CRC) 871 Regeneration of Complex Capital Goods funded by the German Research Foundation (DFG).

\section{References}

[1] R. D. Stieger and H. P. Hodson, "The transition mechanism of highly loaded low-pressure turbine blades," Journal of Turbomachinery, vol. 126, no. 4, pp. 536-543, 2004.

[2] H. P. Hodson and R. J. Howell, "Bladerow interactions, transition, and high-lift aerofoils in low-pressure turbines," Annual Review of Fluid Mechanics, vol. 37, no. 1, pp. 71-98, 2005.

[3] Y.-C. Chow, O. Uzol, J. Katz, and C. Meneveau, "Decomposition of the spatially filtered and ensemble averaged kinetic energy, the associated fluxes and scaling trends in a rotor wake," Physics of Fluids, vol. 17, no. 8, 2005.

[4] F. Soranna, Y.-C. Chow, O. Uzol, and J. Katz, "The effects of inlet guide vane-wake impingement on the boundary layer and the near-wake of a rotor blade," Journal of Turbomachinery, vol. 132, no. 4, Article ID 041016, 13 pages, 2010.

[5] Z. Gete and R. L. Evans, "An experimental investigation of unsteady turbulent-wake/boundary-layer interaction," Journal of Fluids and Structures, vol. 17, no. 1, pp. 43-55, 2003.
[6] L. Hilgenfeld and M. Pfitzner, "Unsteady boundary layer development due to wake passing effects on a highly loaded linear compressor cascade," Journal of Turbomachinery, vol. 126, no. 4, pp. 493-500, 2004.

[7] J. P. Bons, "A review of surface roughness effects in gas turbines," Journal of Turbomachinery, vol. 132, no. 2, Article ID 021004, 2010.

[8] Q. Zhang, S. Woo Lee, and P. M. Ligrani, "Effects of surface roughness and turbulence intensity on the aerodynamic losses produced by the suction surface of a simulated turbine airfoil," Journal of Fluids Engineering, vol. 126, no. 2, pp. 257-265, 2004.

[9] W. J. Devenport, J. K. Staubs, and A. L. Stewart, "Sound radiation from real airfoils in turbulence," Journal of Sound and Vibration, vol. 329, no. 17, pp. 3470-3483, 2010.

[10] H.-W. D. Chiang and R. E. Kielb, "An analysis system for blade forced response," Journal of Turbomachinery, vol. 115, no. 4, pp. 762-770, 1993.

[11] K. Ekici, R. E. Kielb, and K. C. Hall, "Aerodynamic asymmetry analysis of unsteady flows in turbomachinery," Journal of Turbomachinery, vol. 132, no. 1, Article ID 011006, 2010.

[12] S. Hohenstein, J. Aschenbruck, and J. Seume, "Aerodynamic effects of non-uniform surface roughness on a turbine blade," in Proceedings of the ASME Turbo Expo : Turbine Technical Conference and Exposition (GT '13), San Antonio, Tex, USA, June 2013.

[13] C. Lietmeyer, "Optimal application of riblets on compressor blades and their contamination behavior," Journal of Turbomachinery, vol. 135, no. 1, Article ID 011036, 2012.

[14] J. Hourmouziadis, "Aerodynamic design of low pressure turbines," 1989.

[15] M. Raffel, C. E. Willert, S. T. Wereley, and J. Kompenhans, Particle Image Velocimetry: A Practical Guide, Experimental Fluid Mechanics Series, Springer, 2007, http://books.google.de/ books?id=WH5w2TvK2bQC.

[16] D. Garcia, "A fast all-in-one metho d for automated postprocessing of PIV data," Experiments in Fluids, vol. 50, no. 5, pp. 1247-1259, 2010.

[17] Q. Zhang, P. M. Ligrani, and S. W. Lee, "Determination of rough-surface skin friction coefficients from wake profile measurements," Experiments in Fluids, vol. 35, no. 6, pp. 627-635, 2003.

[18] L. Graftieaux, M. Michard, and G. Nathalie, "Combining PIV, POD and vortex identification algorithms for the study of unsteady turbulent swirling flows," Measurement Science and Technology, vol. 12, no. 9, pp. 1422-1429, 2001.

[19] K. Mulleners and M. Raffel, "The onset of dynamic stall revisited," Experiments in Fluids, vol. 52, no. 3, pp. 779-793, 2012. 

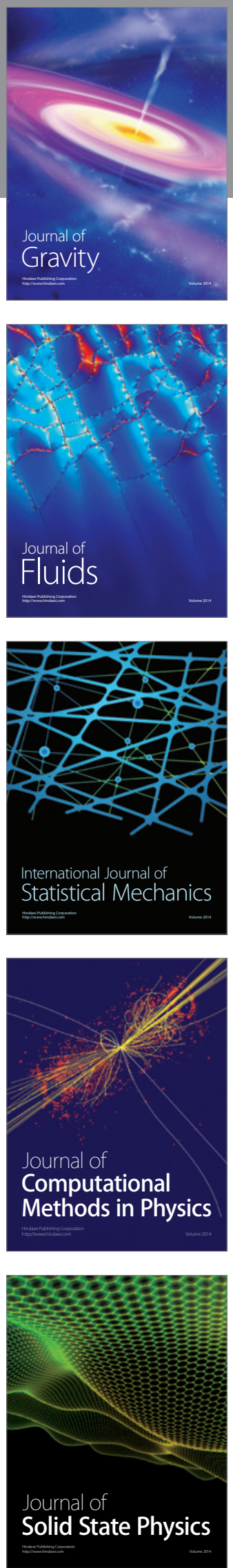

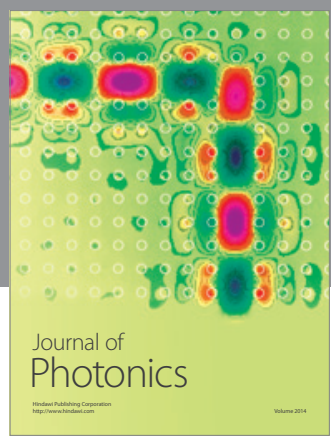

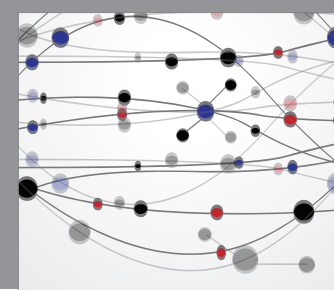

The Scientific World Journal

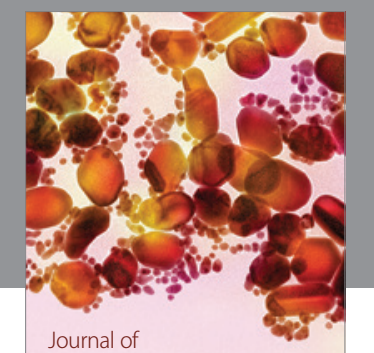

Soft Matter
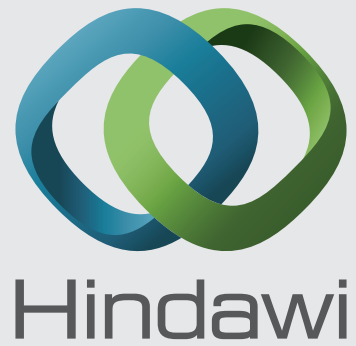

Submit your manuscripts at

http://www.hindawi.com
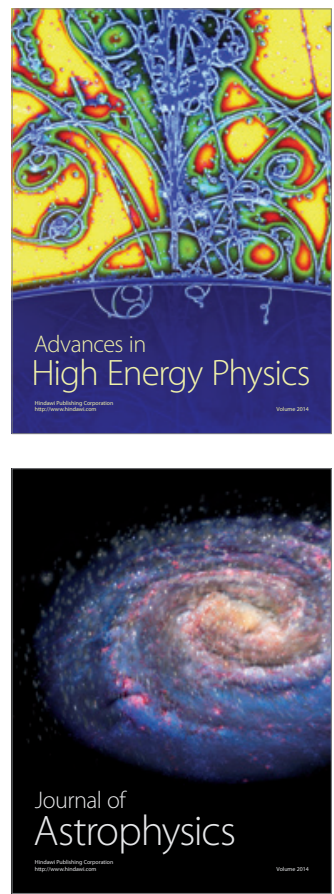
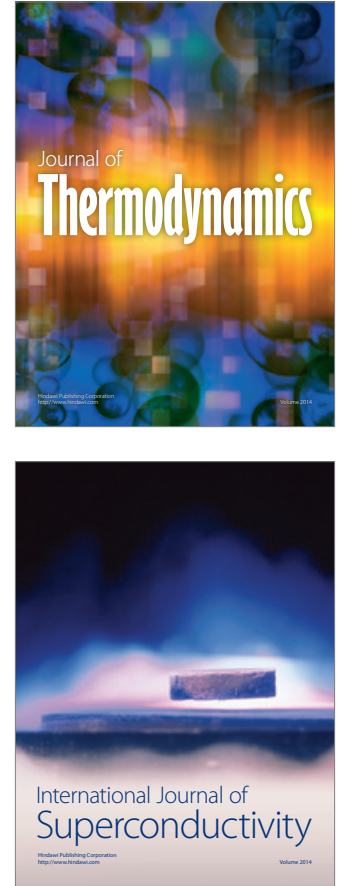
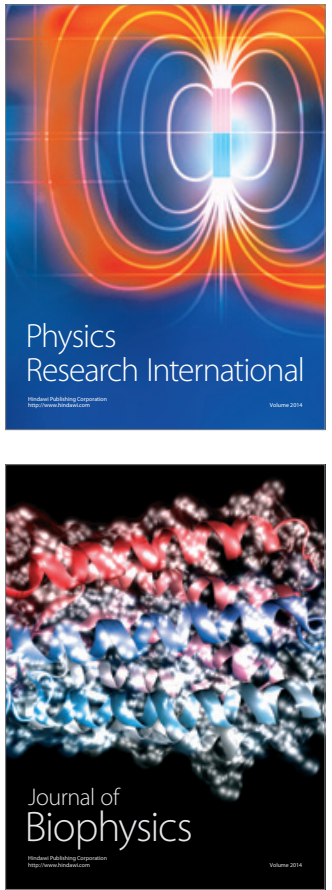
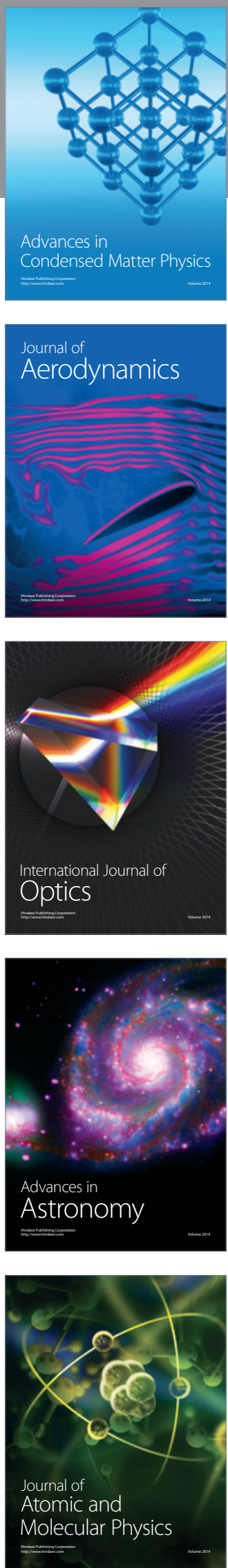